\title{
Plasma concentrations of NOX4 are predictive of successful liberation from mechanical ventilation and 28-day mortality in intubated patients
}

\author{
Yoonki Hong ${ }^{1}$, Seongji Woo ${ }^{2}$, Youngmi Kim², Jae Jun Lee ${ }^{2}$, Ji Young Hong ${ }^{2,3,4}$ \\ ${ }^{1}$ Department of Internal Medicine, Kangwon National University, Chuncheon, Republic of Korea; ${ }^{2}$ Institute of New frontier Research, Hallym \\ University College of Medicine, Republic of Korea; ${ }^{3}$ Division of Pulmonary and Critical Care Medicine, Department of Medicine, Chuncheon \\ Sacred Heart Hospital, Hallym University Medical Center, Chuncheon, Gangwon-do, Republic of Korea; ${ }^{4}$ Lung Research Institute of Hallym \\ University College of Medicine, Chuncheon, Republic of Korea \\ Contributions: (I) Conception and design: JY Hong; (II) Administrative support: Y.Hong; (III) Provision of study materials or patients: S Woo; (IV) \\ Collection and assembly of data: : S Woo, Y Kim; (V) Data analysis and interpretation: JY Hong ,Y Hong, JJ Lee; (VI) Manuscript writing: All \\ authors; (VII) Final approval of manuscript: All authors. \\ Correspondence to: Ji Young Hong, MD, PhD. Assistant Professor, Division of Pulmonary and Critical Care Medicine, Department of Medicine, \\ Chuncheon Sacred Heart Hospital, Hallym University Medical Center, 77, Sakju-ro, Chuncheon-si, Gangwon-do 200-704, Republic of Korea. \\ Email: mdhong@hallym.or.kr.
}

Background: Nicotinamide adenine dinucleotide phosphate (NADPH) oxidase (NOX) enzymes play important roles in generating reactive oxygen species (ROS); in particular, NOX4 plays a distinct role in regulating lung inflammation and apoptosis.

Methods: We determined whether plasma NOX4 level can be used as a prognostic biomarker to guide weaning from mechanical ventilation and to predict mortality in intubated patients. Plasma levels of NOX4 were measured at days 1 (NOX4 D1) and 7 (NOX4 D7) after initiation of mechanical ventilation in 184 patients.

Results: With increase in day 7 NOX4 quartile, the success of weaning tended to decrease and 28-day mortality tended to increase. On multivariate logistic regression, Acute Physiology, Age, Chronic Health Evaluation II (APACHE II) [odds ratio (OR): 1.10; 95\% CI, 1.02-1.18], duration of mechanical ventilation (OR: 1.12; 95\% CI: 1.06-1.18), and NOX4 D7 levels >18.2 ng/mL (OR: 4.40; 95\% CI: 1.91-10.06) were independently associated with weaning failure. Also, Cox-hazard proportional model showed that NOX4 D7 level $>18.2 \mathrm{ng} / \mathrm{mL}$ (hazard ratio [HR], 2.29; 95\% CI, 1.26-4.16), APACHE II (HR: 1.07; 95\% CI: 1.02-1.14), Sequential Organ Failure Assessment (SOFA) (HR: 1.10; 95\% CI: 1.01-1.20) and coexisting cancer (HR: 1.99; 95\% CI, 1.01-3.94), were independently associated with 28-day mortality. The longitudinal trend of NOX4 level varied according to the clinical outcomes.

Conclusions: An increased plasma NOX4 D7 level was associated with weaning failure and 28-day mortality in patients with mechanical ventilation. Our results suggest that NOX4-directed management may lead to improved outcomes in patients with mechanical ventilation.

Keywords: Biomarker; mechanical ventilation; mechanical ventilation weaning; NOX4; mortality

Submitted May 25, 2020. Accepted for publication Sep 18, 2020.

doi: 10.21037/atm-20-4252

View this article at: http://dx.doi.org/10.21037/atm-20-4252 


\section{Introduction}

Extubation failure is associated with high mortality and prolonged intensive care unit (ICU) stay $(1,2)$. Prolonged use of ventilator leads to various complications including ventilator-associated pneumonia, lung barotrauma, and muscle weakness; therefore, it is ideal to wean the patients from the ventilator as soon as possible (3-5).

Several studies have investigated the predictors of successful extubation; however, the sensitivity and specificity of these factors have been largely inconsistent $(6,7)$. Even $2 \mathrm{~h}$ spontaneous breathing test (SBT), which is the current weaning practice, cannot completely predict successful extubation $(8,9)$. The development of readily available point-of-care assays that can help predict weaning success or mortality is of immense clinical relevance.

Nicotinamide adenine dinucleotide phosphate (NADPH) oxidases (NOXs) are enzymes that generate reactive oxygen species (ROS); the role of NOXs has been studied in the context of several lung diseases (10). NOX4 was shown to be associated with lung inflammation and lung permeability (11). Oxidative stress plays a significant role in ventilator-and sepsis-induced diaphragm dysfunctions $(12,13)$. In septic conditions, NOXs enzymes produced by skeletal muscle fiber were shown to increase ROS production $(14,15)$. The mechanism of diaphragm weakness involves mitochondrial ROS production induced by pathogen-associated molecular patterns and dangerassociated molecular patterns, which promotes degradation of sarcomeric proteins via calpains and upregulates the autophagy process (12).

However, the detailed mechanism of NOX4 in ventilator-induced lung inflammation and injury needs to be explored in further studies. Till date, no studies have assessed the association between plasma NOX4 level and clinical outcomes of critical ill patients with mechanical ventilation.

Based on the hypothesis that plasma NOX4 may predict clinical outcomes such as successful weaning from mechanical ventilation and 28-day mortality, we performed serial assessment of plasma NOX4 levels in intubated patients.

We present the following article in accordance with the Strengthening the Reporting of Observational studies in Epidemiology (STROBE) reporting checklist (available at http://dx.doi.org/10.21037/atm-20-4252).

\section{Methods}

\section{Study design and subjects}

The study was conducted at the comprehensive ICU of Chuncheon Sacred Heart Hospital. Patients who were initiated on mechanical ventilation at ICU admission between July 2017 and March 2019 were prospectively recruited and followed up. The exclusion criteria were: (I) age $<18$ years; (II) initiation of mechanical ventilation 48 hours after ICU admission; (III) presence of neuromuscular disease such as amyotrophic lateral sclerosis; (IV) inability to provide informed consent; (V) duration of mechanical ventilation $<7$ days.

Out of the 542 evaluated patients, 358 were excluded because of age criteria $(n=15)$, intubation initiated 48 hours after ICU admission $(n=203)$, presence of neuromuscular disease $(\mathrm{n}=5)$, inability to obtain consent $(\mathrm{n}=11)$, and duration of mechanical ventilation $<7$ days $(\mathrm{n}=124)$. Thus, 184 patients were enrolled in this study (Figure 1). This study was conducted in accordance with the Declaration of Helsinki (as revised in 2013) and harmonized Tripartite Guidelines for Good Clinical Practice from the International Conference on Harmonization. The study was approved by the local ethics committee (IRB number: 2017-47) and informed consent was obtained from each participant.

\section{Data collection}

Data pertaining to baseline demographic variables, comorbid diseases, indication for intubation, and clinical variables were collected. Severity of illness at ICU admission was assessed using the Acute Physiology and Chronic Health Evaluation (APACHE II) score and Sequential Organ Failure Assessment (SOFA) score. APACHE score includes 12 physiological variables, age and previous health status and can give a single score with a maximal of 71 . The SOFA score uses the severity of six organ system (liver, lung coagulation, cardiovascular, renal and neurologic system) and offers a final score from 6 to 24 . Type II diabetes, hypertension, chronic renal failure, history of coronary heart disease, heart failure, cerebrovascular disease, and malignancy were evaluated. Each comorbid condition is assigned a weight ranging from 1 to 6 based on the relative mortality risk and Charlson comorbidity index was calculated by totaling the assigned weight for each 


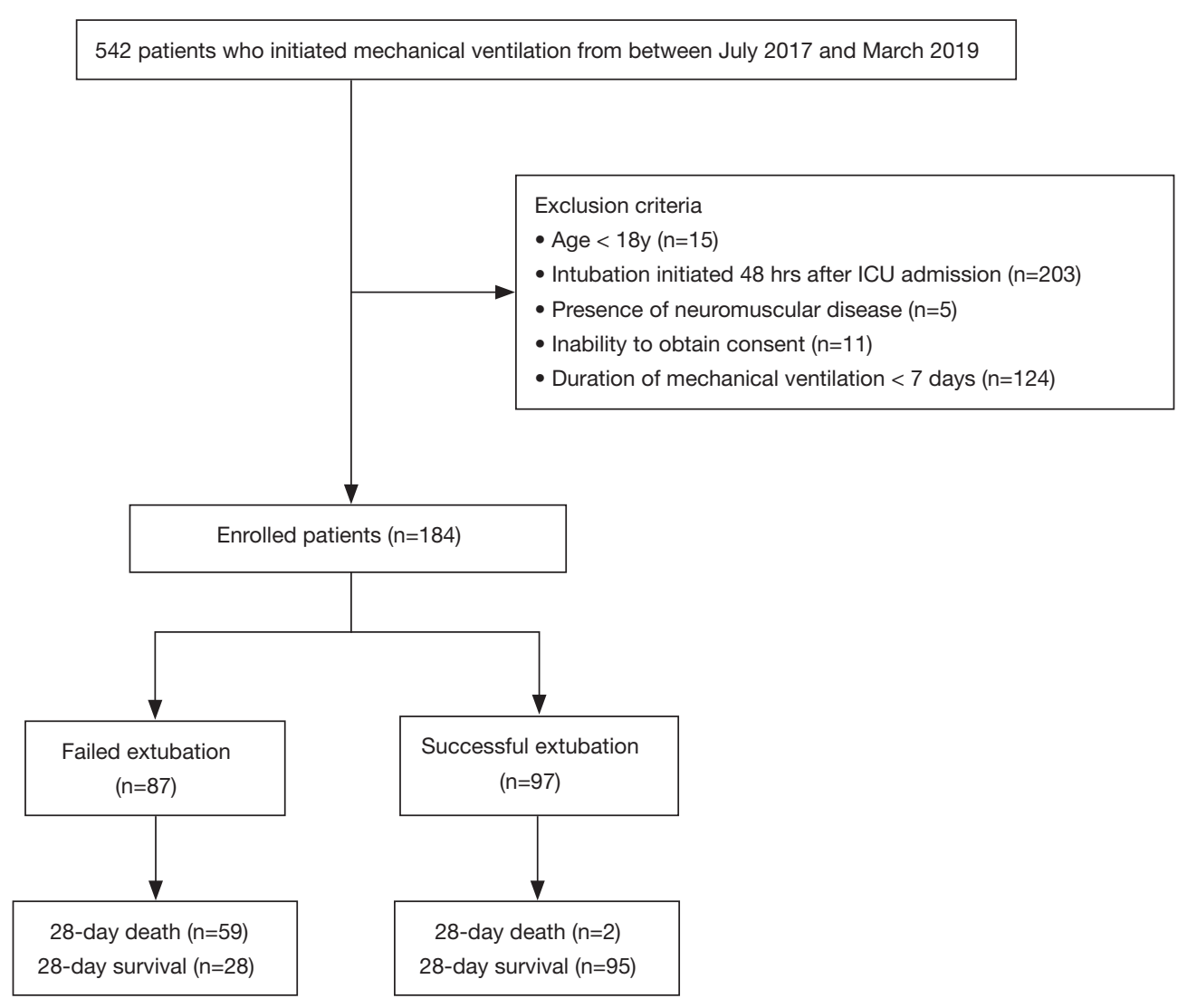

Figure 1 Flow chart of the study population.

comorbid condition (16). Glascow Coma Scale (GCS) score is a neurologic scale to assess the patient's consciousness in the range from 3 to 15 . Clinical outcomes included weaning success in 3 weeks and 28-day mortality.

The ICU attending physicians performed daily weaning assessment after enrollment; patients who qualified the following criteria were considered to have passed the weaning assessment: (I) improvement or resolution of the underlying condition; (II) $\mathrm{PaO}_{2}>60 \mathrm{mmHg}$ at $\mathrm{FiO}_{2}<0.4$ and positive end-expiratory pressure (PEEP) $\leq 5 \mathrm{cmH}_{2} \mathrm{O}$ along with other clinical criteria; (III) GCS score $>13$. All patients who passed weaning assessment underwent 2-hour SBT wherein the patients were placed on spontaneous mode of weaning with low pressure support $\left(8 \mathrm{cmH}_{2} \mathrm{O}\right)$ and zero PEEP with the same $\mathrm{FiO}_{2}(<40 \%)$ for at least $2 \mathrm{~h}$. SBT was passed if patients did not develop any of the following signs during the 2 hour-SBT: respiratory rate $>35$ breaths per minute; arterial oxygen saturation $<90 \%$; heart rate $>140$ beats $/$ min or systolic blood pressure $>180$ or $<90 \mathrm{mmHg}$; sustained increase or decrease in heart rate
$>20 \%$, or signs of respiratory distress such as agitation and diaphoresis.

Weaning failure was defined as either the failure of SBT or the need for reintubation within 48 hours of extubation. Rapid shallow breathing index (RSBI) score was obtained by dividing respiratory rate by tidal volume and was measured when the patients were presumed ready for extubation. The scoring was conducted by two clinicians with more than 5 years of experience in ICU and the investigators were blinded to patient identity.

\section{Plasma NOX4 measurement}

Plasma was obtained within 24 hours from initiation of mechanical ventilation (D1) and at day 7 (D7). After supine rest for at least $10 \mathrm{~min}$, fasting blood samples were collected and centrifuged at 2,000 rpm for $15 \mathrm{~min}$ at room temperature. Plasma samples were stored at $-80{ }^{\circ} \mathrm{C}$ until further processing and assayed using Human NOX4 (NADPH oxidase 4) ELISA kit (MyBiosource, USA). 


\section{Sample size determination}

We calculated that 149 patients per group were required to have a $90 \%$ chance of detecting as significant at the $5 \%$ level a mean \pm SD difference of $15 \pm 40 \mathrm{pg} / \mathrm{mL}$ in plasma NOX4 between groups.

\section{Statistical analysis}

Categorical variables are reported as frequency (percentage) and continuous variables are expressed as median [interquartile range (IQR)]. Categorical variables were compared using Chi-square test while continuous variables were compared using Mann-Whitney $U$ test. The nonparametric Wilcoxon signed-rank test was used for comparisons between D1 and D7 measurements. Patients were stratified into groups according to low or high NOX4 concentrations using the median value for each as the cutoff point (D1 NOX4 level: $16.8 \mathrm{ng} / \mathrm{mL}$, D7 NOX4 level: $18.2 \mathrm{ng} / \mathrm{mL})$.

Multivariate logistic regression analysis was performed to evaluate the risk factors for weaning failure. The effect of plasma NOX4 level was assessed after adjusting for confounding factors and important risk factors. Variables that were associated with $\mathrm{P}$ values $<0.1$ in the univariate analysis were incorporated in the multiple logistic regression model. Kaplan-Meier survival curves were constructed for the 28-day period after ICU admission. Cumulative survival rates were compared using the log-rank test; the association between D7 NOX4 level and probability of 28day mortality was assessed using Cox regression analysis. The non-parametric Wilcoxon signed-rank test was used for paired comparisons of the longitudinal change between D1 and D7 NOX4 levels.

Statistical analyses were performed using SPSS v. 20.0 (SPSS Inc., USA) and Prism 5.1 (Graphpad software, USA). Two-tailed $\mathrm{P}$ values $<0.05$ were considered indicative of statistical significance.

\section{Results}

\section{Characteristics of the study population}

The demographic and baseline characteristics of the study population are summarized in Table 1. A total of 184 patients were enrolled; of these, only 97 patients were successfully extubatedwithin 3 weeks and 61 patients died within 28 days from ICU admission. Patients with extubation failure were significantly older (76 vs. 69 years, $\mathrm{P}=0.003)$. The APACHE
II score and SOFA score in the failed extubation group were significantly higher than that in the successful extubation group \{median (IQR), APACHE: 23 [20-28] vs. 19 [15-23]; $\mathrm{P}<0.001$, SOFA: 9 [7-12] vs. 6 [5-9]; $\mathrm{P}<0.001$; ; however, no significant between-group difference was observed with respect to Charlson comorbidity index $[2(1,3)$ vs. $2(1,3)$, $\mathrm{P}=0.225]$. The indications for intubation and the proportion of patients with pneumonia at admission were not different between the two groups.

The duration of mechanical ventilation (MV) in the failed extubation group was longer than that in the successful extubation group. The failed extubation group showed higher 28-day mortality and ICU mortality than the successful extubation group. In addition, plasma NOX4 levels on D7 in the failed extubation group were significantly higher than those in the successful extubation group.

\section{NOX4 level and clinical outcomes}

NOX4 levels from D1 and D7 were analyzed to assess the association with weaning failure. Logistic regression analysis showed that APACHE score, duration of mechanical ventilation, and D7 plasma NOX4 levels $>18.2 \mathrm{ng} / \mathrm{mL}$ were independently associated with weaning failure (Table 2). Higher D7 NOX4 level $>18.2 \mathrm{ng} / \mathrm{mL}$ was associated with increased odds of weaning failure in both univariate (odds ratio [OR], 3.54; 95\% CI, 1.93-6.51; $\mathrm{P}<0.001)$ and multivariate analyses $(\mathrm{OR}, 4.40 ; 95 \% \mathrm{CI}$, 1.91-10.06; $\mathrm{P}<0.001)$. Higher D1 NOX4 level was not significantly associated with increased odds of weaning failure $(\mathrm{P}=0185)$.

We disaggregated the study population according to quartiles of D7 NOX4 levels and assessed their association with success of weaning and 28-day mortality (Figure 2). With increase in NOX4 quartile, the success of weaning tended to decrease and 28-day mortality tended to increase. As shown, each trend was statistically significant.

Kaplan-Meier survival analysis showed that the cumulative survival rate was significantly lower in patients with D7 NOX4 levels $>18.2 \mathrm{ng} / \mathrm{mL}(53.8 \%$ vs. $80.2 \%$, respectively; $\mathrm{P}<0.001$ ) (Figure 3). Using Cox regression analysis, the ability of D7 NOX4 level and several variables to predict mortality was evaluated (Table 3). Coexisting cancer (HR, 1.99; 95\% CI, 1.01-3.94), APACHE II score (HR, 1.07; 95\% CI,1.02-1.14), SOFA score (HR, 1.10; 95\% CI, 1.01-1.20) and D7 NOX4 >18.2 ng/mL (HR, 2.29; 95\% CI, 1.26-4.16) were independently associated with 28-day mortality. 
Table 1 Demographics and baseline characteristics of the study population

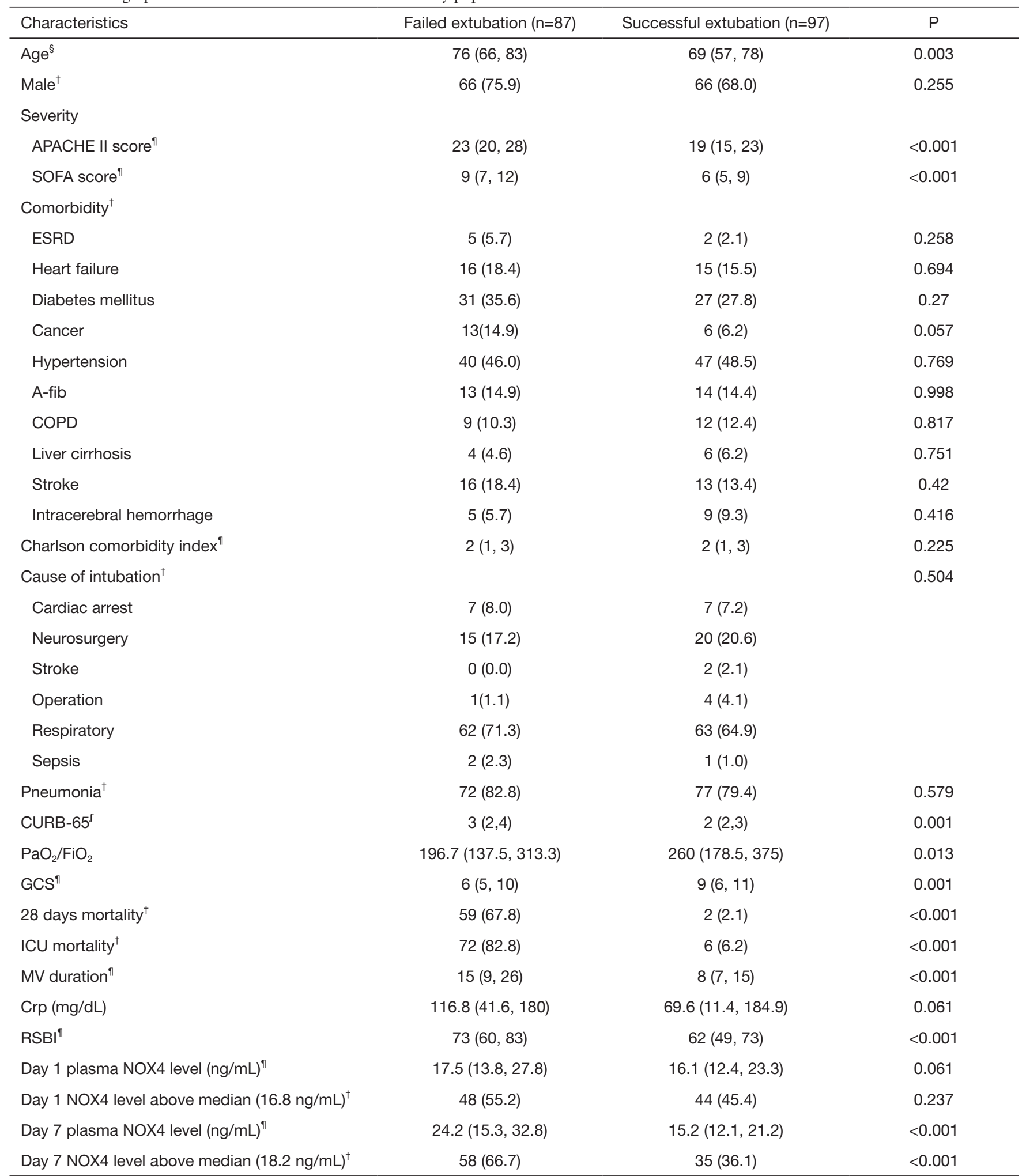

\footnotetext{
", median (interquartile range); ${ }^{\dagger}$, frequency $(\%) ;{ }^{\S}$, median (range); ${ }^{\varsigma}$, limited to patients with pneumonia. APACHE II, Acute Physiology, Age, Chronic Health Evaluation II; SOFA, Sequential Organ Failure Assessment; ESRD, end stage renal disease; GCS, Glasgow Coma Scale ICU, intensive care unit; RSBI, rapid shallow breathing index.
} 
Table 2 Results of logistic regression model showing odds of weaning failure within 3 weeks $(n=184)$

\begin{tabular}{|c|c|c|c|c|}
\hline & \multicolumn{2}{|c|}{ Univariate analysis } & \multicolumn{2}{|c|}{ Multivariate analysis } \\
\hline RSBI & $1.03(1.00,1.04)$ & 0.003 & $1.01(1.13,1.03)$ & 0.602 \\
\hline Age & $1.04(1.01,1.06)$ & 0.003 & $1.03(0.99,1.06)$ & 0.131 \\
\hline APACHE II score & $1.15(1.09,1.22)$ & $<0.001$ & $1.10(1.02,1.18)$ & 0.009 \\
\hline MV days & $1.09(1.05,1.13)$ & $<0.001$ & $1.12(1.06,1.18)$ & $<0.001$ \\
\hline Day 1 NOX4 level above median (16.8 ng/mL) & $1.48(0.83,2.65)$ & 0.185 & & \\
\hline Day 7 NOX4 level above median (18.2 ng/mL) & $3.54(1.93,6.51)$ & $<0.001$ & $4.40(1.91,10.06)$ & $<0.001$ \\
\hline
\end{tabular}

RSBI, rapid shallow breathing index; APACHE, Acute Physiology and Chronic Health Evaluation II; SOFA, Sequential Organ Failure Assessment; MV, mechanical ventilation; NOX4, NADPH oxidase 4; OR, odds ratio.

A

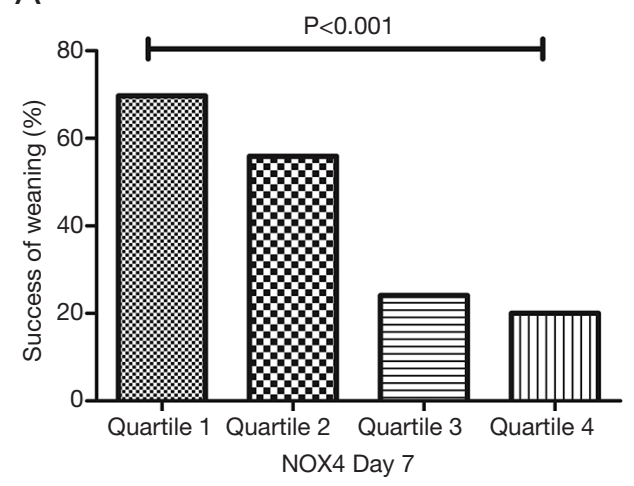

B

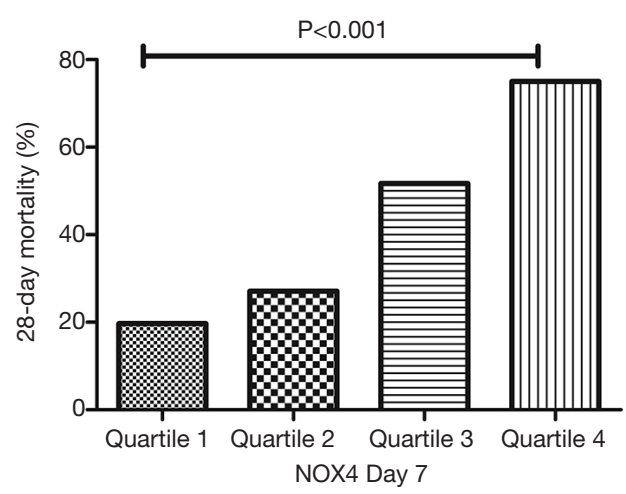

Figure 2 Day 7 NOX4 level and clinical outcomes. (A) Success of weaning within 3 weeks according to day 7 NOX4 quartiles; (B) 28 -day mortality according to day 7 NOX4 quartiles (n=184). NOX4, NADPH oxidase 4.

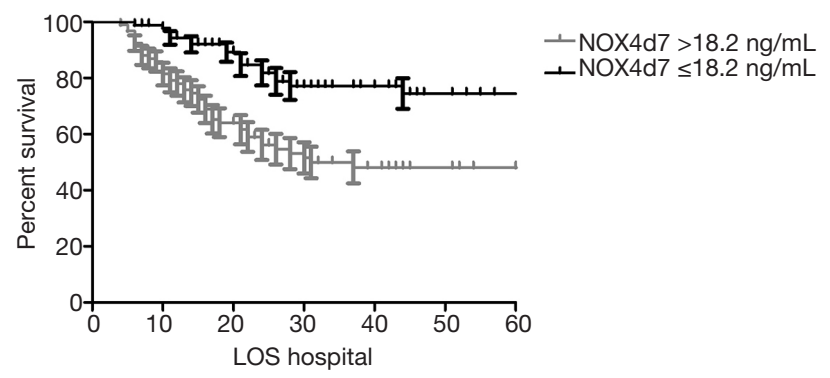

Figure 3 Kaplan-Meier survival curves in 28 days according to day 7 NOX4 levels. Kaplan-Meier survival analysis showed that the 28-day mortality of patients with plasma NOX4 level $>18.2 \mathrm{ng} / \mathrm{mL}$ was higher than that of patients with plasma NOX4 level $\leq 18.2 \mathrm{ng} / \mathrm{mL}$. NOX4, NADPH oxidase 4.

\section{Trend of NOX4 levels between D1 and D7 according to weaning failure and 28-day mortality}

We analyzed the longitudinal trend of NOX4 level according to clinical outcomes in the study population (Figure 4). In the failed extubation group, the baseline level of NOX4 significantly increased between D1 and D7 (P<0.001; median at D1: $17.5 \mu \mathrm{g} / \mathrm{mL}$; IQR 13.8-27.8; median at D7: $24.2 \mu \mathrm{g} / \mathrm{mL}$; IQR $15.3-32.8)$ but not in the successful extubation group $(\mathrm{P}=0.081$; median at $\mathrm{D} 1$ : $16.1 \mu \mathrm{g} / \mathrm{mL}$; IQR 12.4-23.3; median at D7: $15.1 \mu \mathrm{g} / \mathrm{mL}$; IQR 12.2-20.8).

Similarly, in the 28-day death group, the level of NOX4 significantly increased between D1 and D7 ( $\mathrm{P}=0.001$; 
Table 3 Results of Cox regression analysis for survival prediction in intubated patients $(\mathrm{n}=184)$

\begin{tabular}{lccc}
\hline Variable & Hazard ratio & $95 \% \mathrm{Cl}$ & \\
\hline Cancer & 1.99 & $1.01,3.94$ & 0.045 \\
Age & 1.02 & $0.99,1.04$ & 0.133 \\
APACHE II score & 1.07 & $1.02,1.14$ & 0.006 \\
SOFA score & 1.1 & $1.01,1.20$ & 0.029 \\
Day 1 NOX4 level above median $(16.8 \mathrm{ng} / \mathrm{mL})$ & 0.81 & $0.45,1.48$ & 0.497 \\
Day 7 NOX4 level above median $(18.2 \mathrm{ng} / \mathrm{mL})$ & 2.29 & $1.26,4.16$ & 0.006 \\
\hline
\end{tabular}

APACHE, Acute Physiology and Chronic Health Evaluation II; SOFA, Sequential Organ Failure Assessment; NOX4, NADPH oxidase 4.
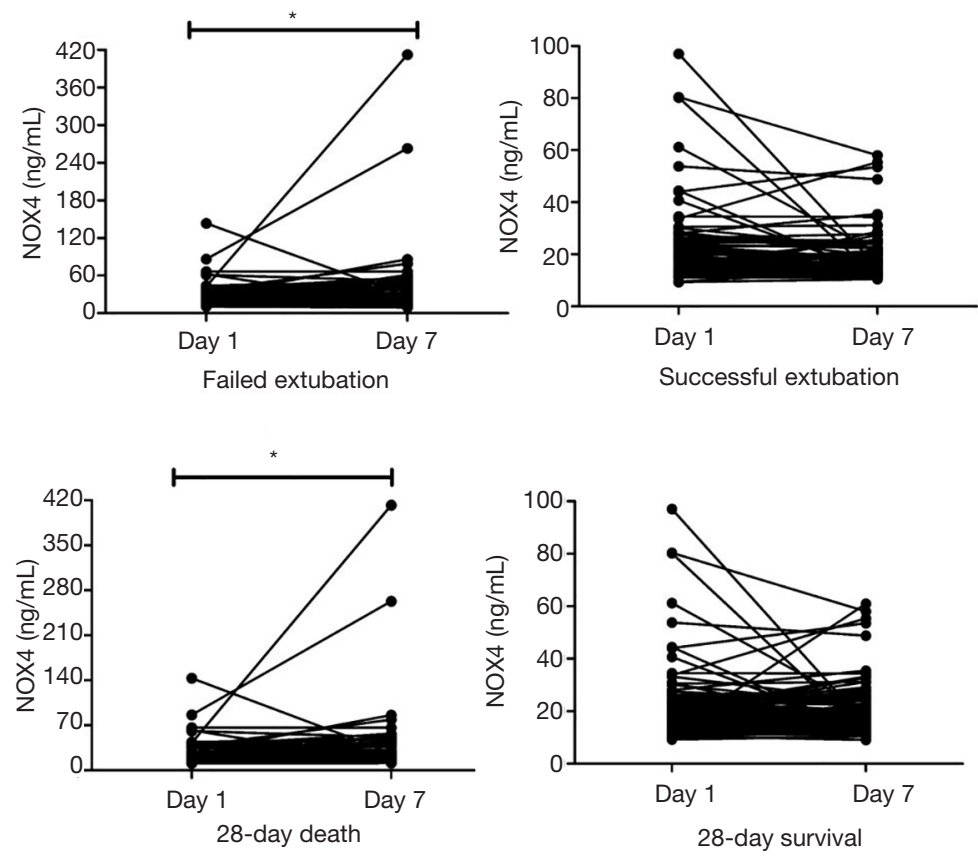
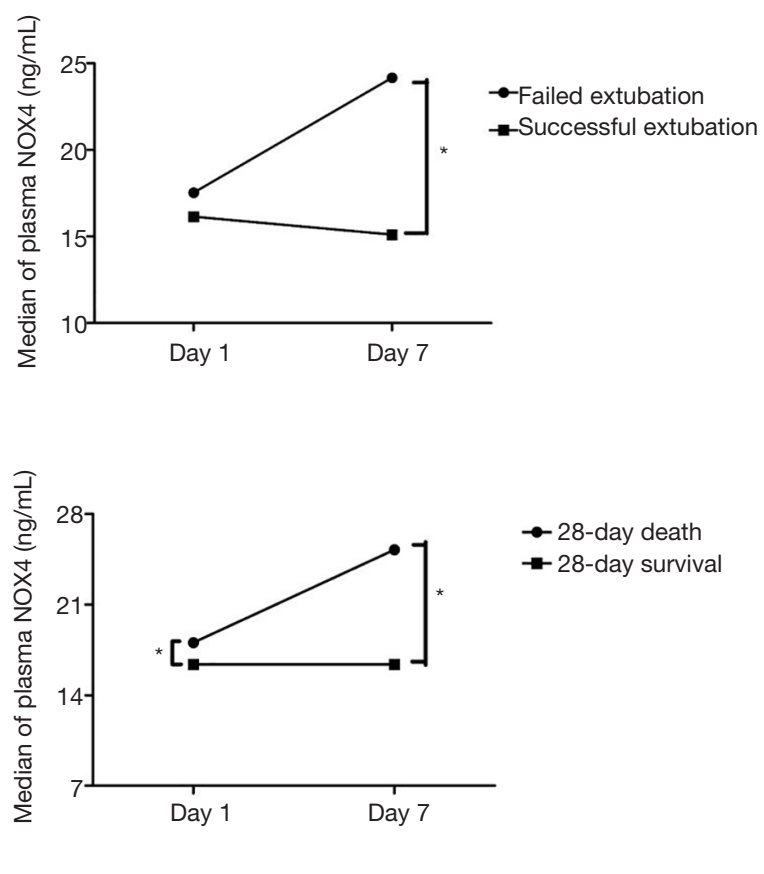

Figure 4 Longitudinal trend of plasma NOX4 level between day 1 and day 7 from start of mechanical ventilation according to weaning outcome (A) and 28-day mortality (B). *, $\mathrm{P}<0.05$. NOX4, NADPH oxidase 4.

median at D1: $18.1 \mu \mathrm{g} / \mathrm{mL}$; IQR 13.8-34.4; median at D7: $25.2 \mu \mathrm{g} / \mathrm{mL}$; IQR 15.9-41.7); however, this phenomenon was not observed in the 28-day survival group $(\mathrm{P}=0.744$; median at D1: $16.4 \mu \mathrm{g} / \mathrm{mL}$; IQR $12.5-22.7$; median at D7: $16.4 \mu \mathrm{g} / \mathrm{mL}$; IQR 12.4-22.0).

\section{Discussion}

Several indices have been used to predict successful extubation such as RSBI and maximal inspiratory pressure (MIP) (17-20). However, the reported efficacy of these parameters for predicting successful extubation has been inconsistent; in addition, assessment of these indices requires a special device, which is a limitation (21-24).

McConville et al. reported that improvement in the underlying disease process is superior to general rules regarding readiness for initiation of SBT (25). Therefore, more objective measures are required to guide the recovery of underlying cause of respiratory failure and to predict the likelihood of extubation.

In the current study, we investigated the risk factors related to extubation failure and 28-day mortality in patients who initiated mechanical ventilation. In accordance with previous studies, our study showed that prolonged 
duration of ventilation before extubation was a risk factor for extubation failure (24) and malignancy was a significant risk factor for in-hospital mortality in patients receiving mechanical ventilation support $(26,27)$. The most important finding was that NOX4 level may help predict successful weaning off from mechanical ventilation and 28-day mortality.

Biologically, NOX4 is believed to play a role in endothelial signal transduction, cytoskeletal reorganization, and apoptosis of endothelial cells $(13,28)$. NOX4 is upregulated in several pulmonary diseases including tuberculous fibrosis, idiopathic pulmonary fibrosis, and lung cancer $(29,30)$. The previous studies demonstrated that NOX4 was shown to play a distinct role in ventilatorinduced lung injury and Pseudomonas aeruginosa-induced lung inflammation (11,31).

While Canugovi et al. reported that NOX4 expression and ROS levels increase with age (32), our data showed no significant correlation between the plasma NOX4 level and age (D1 NOX4: $r=-0.015, \mathrm{P}=0.839$, D7 NOX4: $\mathrm{r}=0.069$, $\mathrm{P}=0.355$ ). Patients with extubation failure were significantly older than those with extubation success. However, we found that NOX4 levels $>18.2 \mathrm{ng} / \mathrm{mL}$ on D7 showed an independent association with extubation failure and 28-day mortality after adjusting for age and other important clinical variables. Also, the Kaplan-Meier survival curves and Coxhazard proportional model supported the association between higher D7 NOX4 level and 28-day mortality.

To the best of our knowledge, this is the first study that investigated plasma NOX4 levels in critically ill patients. Despite the complex biological mechanisms associated with NOX4, we found obvious clinical relevance of NOX4 levels in our patients.

Our data demonstrated a positive correlation between plasma NOX4 level and the severity markers such as APACHE II score and SOFA score (APACHE II, $\gamma=0.220$, $\mathrm{P}=0.003$; SOFA, $\gamma=0.316, \mathrm{P}<0.001)$. These results suggest a correlation of NOX4 level with systemic inflammation and disease severity. Interestingly, ROC curve analysis showed that the area under the curve of D7 NOX4 level was similar with that of APACHE II or SOFA (weaning failure; NOX4: 0.721, APACHE II: 0.722, SOFA: 0.720, 28-day mortality; NOX4: 0.719, APACHE II: 0.699, SOFA: 0.722) (Figure S1).

In multivariate analysis of our data, while APACHE II score was independently associated with both clinical outcomes, SOFA score was independently associated with only 28-day mortality. Similarly, the previous studies showed that APACHE II scoring system predicted early weaning as well as mortality $(33,34)$. While Matic et al. reported that an APACHE II score of $<20$ indicated greater success in weaning the patient from the ventilator (35), some studies found that APACHE II failed to predict successful weaning from mechanical ventilation $(36,37)$. A multicenter study conducted in surgical ICUs reported that SOFA score was a risk factor of extubation failure (38). The discrepancy between studies may be explained by heterogeneity with respect to subjects and the size of the study population.

As shown in Figure 4, we observed that NOX4 levels were not significantly different between the failed extubation group and the successful extubation group at initial assessment, but the difference between the two groups was particularly marked at D7 due to increasing trend in the failed extubation group. Interestingly, similar results were observed between survivors and nonsurvivors. Follow up of NOX4 levels to identify trends may help predict the clinical outcomes in intubated patients.

Use of NOX4 level as a prognostic biomarker may be more accurate because it is significantly related to both weaning outcome and mortality. This study is the first clinical study that employed human blood samples to evaluate the relationship between plasma NOX4 level and clinical outcomes including weaning failure and 28-day mortality.

However, some limitations of our study should be considered while interpreting the results. First, this study was conducted at a single university-affiliated hospital and the study population was relatively small. Therefore, further validation is required before our results can be generalized to other clinical settings. Second, measurement of NOX4 level was done at D1 and D7 of ICU admission. However, analysis at multiple time points may be required to determine the most appropriate measurement time-point to predict clinical outcomes. Third, invitro experimental data to support the potential prognostic utility of plasma NOX4 level is lacking. The exact underlying mechanism by which NOX4 activation contributes to extubation failure and mortality needs to be explored in further studies.

In conclusion, this study showed that higher D7 plasma NOX4 level was significantly associated with weaning failure and 28-day mortality among intubated patients. Serial measurements of plasma NOX4 level may help predict the clinical outcomes. Further larger studies are needed to determine whether plasma NOX4 level may be a 
potential prognostic biomarker in patients with mechanical ventilation.

\section{Acknowledgements}

Funding: This research was supported by the Bio \& Medical Technology Development Program of the National Research Foundation (NRF) funded by the Korean government (MSIT) (NRF-2017M3A9E8033225 to JY Hong) and was supported by the National Research Foundation of Korea Grant funded by Korean Government (NRF 2020R1A2C1011455 to JY Hong).This study was supported by 2018 Research Grant from Kangwon National University and 2018 Research Grant from Kangwon National University Hospital (to Y Hong). The funders had no role in study design, data collection and interpretation or preparation of the manuscript.

\section{Footnote}

Reporting Checklist: The authors have completed the STROBE reporting checklist, available at http://dx.doi. org/10.21037/atm-20-4252

Data Sharing Statement: Available at http://dx.doi. org/10.21037/atm-20-4252

Peer Review File: Available at http://dx.doi.org/10.21037/ atm-20-4252

Conflicts of Interest: All authors have completed the ICMJE uniform disclosure form (available at http://dx.doi. org/10.21037/atm-20-4252). The authors have no conflicts of interest to declare.

Ethnical Statement: The authors are accountable for all aspects of the work in ensuring that questions related to the accuracy or integrity of any part of the work are appropriately investigated and resolved. This study was conducted in accordance with the Declaration of Helsinki (as revised in 2013) and harmonized Tripartite Guidelines for Good Clinical Practice from the International Conference on Harmonization. The study was approved by the local ethics committee (IRB number: 2017-47) and informed consent was obtained from each participant.

Open Access Statement: This is an Open Access article distributed in accordance with the Creative Commons Attribution-NonCommercial-NoDerivs 4.0 International License (CC BY-NC-ND 4.0), which permits the noncommercial replication and distribution of the article with the strict proviso that no changes or edits are made and the original work is properly cited (including links to both the formal publication through the relevant DOI and the license). See: https://creativecommons.org/licenses/by-nc-nd/4.0/.

\section{References}

1. Mancebo J. Weaning from mechanical ventilation. Eur Respir J 1996;9:1923-31.

2. Seymour CW, Martinez A, Christie JD, et al. The outcome of extubation failure in a community hospital intensive care unit: a cohort study. Crit Care 2004;8:R322-7.

3. Cook DJ, Walter SD, Cook RJ, et al. Incidence of and risk factors for ventilator-associated pneumonia in critically ill patients. Ann Intern Med 1998;129:433-40.

4. De Jonghe B, Bastuji-Garin S, Durand MC, et al. Respiratory weakness is associated with limb weakness and delayed weaning in critical illness. Crit Care Med 2007;35:2007-15.

5. Jubran A. Critical illness and mechanical ventilation: effects on the diaphragm. Respir Care 2006;51:1054-61; discussion 62-4.

6. Lessard MR, Brochard LJ. Weaning from ventilatory support. Clin Chest Med 1996;17:475-89.

7. Aboussouan LS, Lattin CD, Anne VV. Determinants of time-to-weaning in a specialized respiratory care unit. Chest 2005;128:3117-26.

8. Esteban A, Alia I, Tobin MJ, et al. Effect of spontaneous breathing trial duration on outcome of attempts to discontinue mechanical ventilation. Spanish Lung Failure Collaborative Group. Am J Respir Crit Care Med 1999;159:512-8.

9. Esteban A, Alia I, Gordo F, et al. Extubation outcome after spontaneous breathing trials with T-tube or pressure support ventilation. The Spanish Lung Failure Collaborative Group. Am J Respir Crit Care Med 1997;156:459-65.

10. Bernard K, Hecker L, Luckhardt TR, et al. NADPH oxidases in lung health and disease. Antioxid Redox Signal 2014;20:2838-53.

11. Fu P, Mohan V, Mansoor S, et al. Role of nicotinamide adenine dinucleotide phosphate-reduced oxidase 


\section{Page 10 of 11}

proteins in Pseudomonas aeruginosa-induced lung inflammation and permeability. Am J Respir Cell Mol Biol 2013;48:477-88.

12. Petrof BJ. Diaphragm Weakness in the Critically Ill: Basic Mechanisms Reveal Therapeutic Opportunities. Chest 2018;154:1395-403.

13. Pendyala S, Gorshkova IA, Usatyuk PV, et al. Role of Nox4 and Nox 2 in hyperoxia-induced reactive oxygen species generation and migration of human lung endothelial cells. Antioxid Redox Signal 2009;11:747-64.

14. Supinski G, Stofan D, Nethery D, et al. Apocynin improves diaphragmatic function after endotoxin administration. J Appl Physiol (1985) 1999;87:776-82.

15. Javeshghani D, Magder SA, Barreiro E, et al. Molecular characterization of a superoxide-generating $\mathrm{NAD}(\mathrm{P}) \mathrm{H}$ oxidase in the ventilatory muscles. Am J Respir Crit Care Med 2002;165:412-8.

16. Moro-Sibilot D, Aubert A, Diab S, et al. Comorbidities and Charlson score in resected stage I nonsmall cell lung cancer. Eur Respir J 2005;26:480-6.

17. Capdevila XJ, Perrigault PF, Perey PJ, et al. Occlusion pressure and its ratio to maximum inspiratory pressure are useful predictors for successful extubation following T-piece weaning trial. Chest 1995;108:482-9.

18. Epstein SK. Etiology of extubation failure and the predictive value of the rapid shallow breathing index. Am J Respir Crit Care Med 1995;152:545-9.

19. Kulkarni AP, Agarwal V. Extubation failure in intensive care unit: predictors and management. Indian J Crit Care Med 2008;12:1-9.

20. Mergoni M, Costa A, Primavera S, et al. [Assessment of various new predictive parameters of the outcome of mechanical ventilation weaning]. Minerva Anestesiol 1996;62:153-64.

21. Salam A, Tilluckdharry L, Amoateng-Adjepong Y, et al. Neurologic status, cough, secretions and extubation outcomes. Intensive Care Med 2004;30:1334-9.

22. Uusaro A, Chittock DR, Russell JA, et al. Stress test and gastric-arterial $\mathrm{PCO} 2$ measurement improve prediction of successful extubation. Crit Care Med 2000;28:2313-9.

23. Del Rosario N, Sassoon CS, Chetty KG, et al. Breathing pattern during acute respiratory failure and recovery. Eur Respir J 1997;10:2560-5.

24. Lee KH, Hui KP, Chan TB, et al. Rapid shallow breathing (frequency-tidal volume ratio) did not predict extubation outcome. Chest 1994;105:540-3.

\section{Hong et al. Plasma NOX4 level as a predictor of clinical prognosis}

25. McConville JF, Kress JP. Weaning patients from the ventilator. N Engl J Med 2012;367:2233-9.

26. Shih CY, Hung MC, Lu HM, et al. Incidence, life expectancy and prognostic factors in cancer patients under prolonged mechanical ventilation: a nationwide analysis of 5,138 cases during 1998-2007. Crit Care 2013;17:R144.

27. Keng LT, Chung KP, Lin SY, et al. Significant Clinical Factors Associated with Long-term Mortality in Critical Cancer Patients Requiring Prolonged Mechanical Ventilation. Sci Rep 2017;7:2148.

28. Hu T, Ramachandrarao SP, Siva S, et al. Reactive oxygen species production via NADPH oxidase mediates TGFbeta-induced cytoskeletal alterations in endothelial cells. Am J Physiol Renal Physiol 2005;289:F816-25.

29. Kim Y, Park SY, Jung H, et al. Inhibition of NADPH Oxidase 4 (NOX4) Signaling Attenuates Tuberculous Pleural Fibrosis. J Clin Med 2019;8:116.

30. Amara N, Goven D, Prost F, et al. NOX4/NADPH oxidase expression is increased in pulmonary fibroblasts from patients with idiopathic pulmonary fibrosis and mediates TGFbeta1-induced fibroblast differentiation into myofibroblasts. Thorax 2010;65:733-8.

31. Ling $\mathrm{X}$, Lou A, Li Y, et al. Losartan regulates oxidative stress via caveolin-1 and NOX4 in mice with ventilatorinduced lung injury. Nan Fang Yi Ke Da Xue Xue Bao 2015;35:1739-44.

32. Canugovi C, Stevenson MD, Vendrov AE, et al. Increased mitochondrial NADPH oxidase 4 (NOX4) expression in aging is a causative factor in aortic stiffening. Redox Biol 2019;26:101288.

33. Dehghani A, Abdeyazdan G, Davaridolatabadi E. An Overview of the Predictor Standard Tools for Patient Weaning from Mechanical Ventilation. Electron Physician 2016;8:1955-63.

34. Carpenè N, Vagheggini G, Panait E, et al. A proposal of a new model for long-term weaning: respiratory intensive care unit and weaning center. Respir Med 2010;104:1505-11.

35. Matic I, Titlic M, Dikanovic M, et al. Effects of APACHE II score on mechanical ventilation; prediction and outcome. Acta Anaesthesiol Belg 2007;58:177-83.

36. Rojek-Jarmuła A, Hombach R, Krzych LJ. APACHE II score cannot predict successful weaning from prolonged mechanical ventilation. Chron Respir Dis 2017;14:270-5.

37. Sanabria A, Gomez X, Vega V, et al. Prediction of prolonged mechanical ventilation in patients in the 
intensive care unit A cohort study. Colomb Med (Cali) 2013;44:184-8.

38. Buppha P, Kusumaphanyo C, Chittawatanarat K. Outcomes and Risk Factors of Extubation Failure: A

Cite this article as: Hong Y, Woo S, Kim Y, Lee JJ, Hong JY. Plasma concentrations of NOX4 are predictive of successful liberation from mechanical ventilation and 28-day mortality in intubated patients. Ann Transl Med 2020;8(21):1376. doi: 10.21037/atm-20-4252
Multicenter Study of the THAI Surgical Intensive Care Units (SICUs). J Med Assoc Thai 2016;99 Suppl 6:S136-44. 